\title{
The Role of Confocal Endomicroscopy in the Diagnosis and Management of Pancreatic Cysts
}

The contribution of the role of endoscopic ultrasound-guided confocal endomicroscopy via needle puncture of pancreatic cysts to evaluate their benign or malignant potential more reliably is of potential high importance for both accurate diagnosis of malignancy, leading to timely surgery. Or, otherwise, definitive confirmation of the benign nature of such a pancreatic lesion, saving the patient from anxiety about progression and costly long-term follow-up. Even though this will not be applicable for broadrange usage, it will be a very helpful additional tool in highly specialised centres.
Authors:
Margaret G. Keane, ${ }^{*}$ Stephen P. Pereira²
1. Johns Hopkins Hospital, Department of Gastroenterology and Hepatology, Baltimore, Maryland, USA
2. Institute for Liver and Digestive Health, University College London, Royal Free Hospital Campus, UK
*Correspondence to stephen.pereira@ucl.ac.uk

Disclosure:

The authors have declared no conflicts of interest.

Received:

23.07.20

Accepted:

21.07.21

Keywords:

Endoscopic ultrasound (EUS), intraductal papillary mucinous neoplasm (IPMN), needle-based confocal laser endomicroscopy ( $\mathrm{nCLE}$ ), pancreatic cyst, serous cystic neoplasm (SCN).

Citation:

EMJ. 2021;6[3]:41-49.

\begin{abstract}
Pancreatic cystic lesions are an increasingly common clinical finding. Current diagnostic techniques cannot reliably differentiate patients with high-risk lesions requiring surgical resection from those that can be safely surveyed or discharged. As a result, some patients may undergo unnecessary surgery with associated morbidity while others enter long-term surveillance with associated healthcare costs. Needle-based confocal laser endomicroscopy enables real time microscopic examination of the epithelial lining of a cyst wall at the time of a standard endoscopic ultrasound examination. The procedure is associated with low rates of adverse events, especially when the probe is loaded into the fine-needle aspiration needle before the procedure and examination times are limited. Needle-based confocal laser endomicroscopy has consistently been shown to have better diagnostic accuracy than cytology, which is often paucicellular and non-diagnostic in pancreatic cystic lesions. Studies have shown that diagnostic accuracy in needle-based confocal laser endomicroscopy is 84-95\% in mucinous lesions and 39-99\% in serous lesions. However, this technology is expensive and its place in diagnostic algorithms remains uncertain. Despite this, health economic analyses in certain health systems have been favourable, largely because of its potential to be able to discharge patients with benign lesions, such as serous cystic neoplasms, from long-term surveillance. Widespread adoption of this technology is unlikely but it has the potential to have an important role in indeterminate pancreatic cystic lesions .
\end{abstract}




\section{INTRODUCTION}

Pancreatic cystic lesions (PCLs) are an increasingly common finding. They are present in 1.2-2.6\% of patients undergoing abdominal $\mathrm{CT}^{1,2}$ up to $13.5 \%$ of patients undergoing an abdominal $\mathrm{MRI},{ }^{3}$ and up to $45.0 \%$ of asymptomatic individuals undergoing magnetic resonance cholangiopancreatography. ${ }^{4}$ PCLs have a broad differential diagnosis, including benign lesions such as serous cystic neoplasms (SCNs) and pseudocysts, as well as premalignant lesions such as mucinous cystic neoplasms (MCNs) and intraductal papillary mucinous neoplasms (IPMNs). ${ }^{5}$ In accordance with international and European guidance, patients with PCLs that are thought to be malignant or at high-risk of malignant transformation are referred for immediate surgical resection. Patients with lowrisk but premalignant cysts are recommended to undergo long-term surveillance. ${ }^{6,7}$

However, current diagnostic tools are imperfect and cannot always differentiate between highand low-risk cysts. As a result, approximately onefifth of patients with completely benign disease undergo unnecessary pancreatic resections annually. ${ }^{8}$ In addition, differentiating premalignant cysts from all other cysts continues to be challenging. As a result, relatively few patients are discharged from follow-up and growing numbers of patients are entering surveillance. This is anxiety-provoking for patients and costly for healthcare systems.
Consequently, there has been an interest in developing novel diagnostic tools that improve the pre-operative assessment of PCL, such as through the needle biopsy forceps, $, 9,10$ molecular fluid markers," and needle-based confocal laser endomicroscopy (nCLE; Figure 1). This review will focus on the utility of nCLE to differentiate pancreatic cyst subtypes and accurately detect malignant lesions. It will also examine the safety profile of this novel technology and I earning curve.

\section{METHODS}

PubMed, EMBASE, and the Cochrane Library were reviewed for studies published in the English language up to $1^{\text {st }}$ July 2020. Medical Subject Headings (MeSH) terms were decided by a consensus of the authors and were "confocal endomicroscopy AND pancreatic neoplasms", and were restricted to the title, abstract, and keywords. Articles that described the use of nCLE in solid pancreatic lesions were excluded. Any study with fewer than three patients was excluded.

All references were screened for potentially relevant studies not identified in the initial literature search. The following seven variables were extracted for each report when available: number of patients, type of cyst, sensitivity, specificity, positive predictive value, negative predictive value, and diagnostic accuracy. The outcomes of 17 papers are presented in the review (Figure 2).

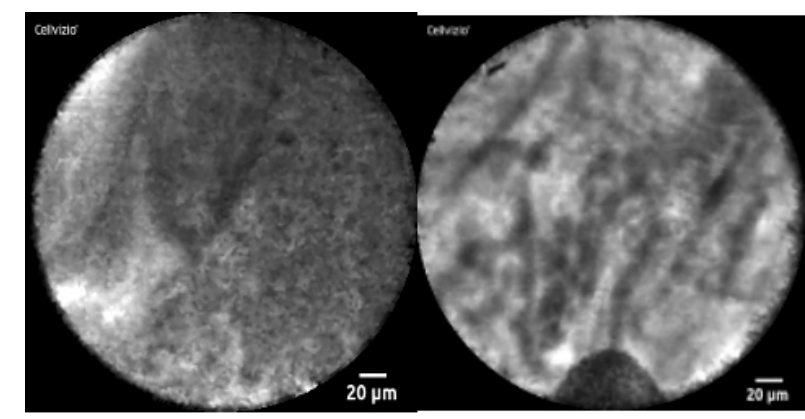

Figure 1: Needle-based confocal laser endomicroscopy pancreatic cyst images.

A) Needle-based confocal laser endomicroscopy image of a superficial vascular network, which is indicative of a serous cystic neoplasm. B) Needle-based confocal laser endomicroscopy image of papillary projections consistent with an intraductal papillary mucinous neoplasm. 




Figure 2: Review schema.

Due to the significant heterogeneity in the studies, a pooled analysis (or a meta-analysis) was not performed.

\section{Procedure}

Confocal laser endomicroscopy is a novel diagnostic device that can provide real time optical histology. nCLE creates an equivalent pathological image via the transmission of a lowpower laser, which be focused on the epithelium of the cyst wall.

The AQ-Flex miniprobe (Cellvizio; Mauna Kea Technologies, Paris, France) can be passed down a 19-gauge fine-needle aspiration (FNA) needle during an endoscopic ultrasound (EUS) examination. The reflected fluorescent light is returned to the operating system, via the probe, to form an image.

To perform an EUS-nCLE examination, the AQFlex probe is loaded into a 19-gauge FNA needle. The PCL is then identified and $2.5 \mathrm{ml}$ of $10 \%$ fluorescein is administered intravenously. Once the cyst is punctured under EUS control, the probe is then advanced until the tip protrudes 2 $\mathrm{mm}$ beyond the needle's bevel and the device is locked at this level. Unlocking the probe allows it to be retracted into the needle, which is used during cyst puncture. To begin scanning, the needle and probe are advanced until in contact with the cyst wall. Pictures and short sequences of video are then recorded for no more than 10 minutes in most cases. The probe can then be removed from the needle and cyst fluid aspiration performed for amylase, carcinoembryonic antigen, cytology, and other assays as required. Antibiotic prophylaxis is recommended for all EUS-nCLE cases.

\section{RESULTS}

\section{Safety and Efficacy}

The first EUS-nCLE study in PCL used a prototype miniprobe. Procedures were undertaken in four centres in the USA. Eighteen patients were included: 16 patients with a PCL and two with a solid pancreatic mass. nCLE images were obtained in $94.4 \%$ but technical challenges were encountered in $33.3 \%$ and post-procedural pancreatitis occurred in $11.1 \%$. The authors concluded that nCLE was a feasible technique to obtain additional clinically relevant information about the cyst. The rate of pancreatitis was higher than expected and therefore the authors recommended modifications such as limiting 
imaging time and not moving the probe along the cyst wall but instead imaging distinct points on the wall. ${ }^{12}$

\section{Defining and Differentiating Cyst Subtypes}

The INSPECT trial explored the diagnostic potential of this technology in PCL. This multicentre study included 66 patients and was conducted over two stages. In the first part of the study, images were compared to final histology and reviewed by a panel of experts that included a gastrointestinal pathologist. The group recognised that the presence of epithelial villous structures could identify pancreatic cystic neoplasms, which included mucinous cystadenomas, IPMNs, and pancreatic adenocarcinoma. In the second part of the study, the presence of epithelial villous structures had a sensitivity, specificity, positive predictive value, and negative predictive value of $59 \%, 100 \%$, $100 \%$, and $50 \%$, respectively. ${ }^{13}$

The DETECT study redefined epithelial villous structures and papillary projections as indicative of mucinous PCL. The study combined cystoscopy using a through-the-needle fibre-optic probe (SpyGlass [Boston Scientific, Marlborough, Massachusetts, USA]) followed by nCLE in a series of 30 patients. The procedure was technically successful in 97\%, with one probe exchange failure. The sensitivity of cystoscopy and nCLE was $71 \%$ and $77 \%$, respectively, but increased to $93 \%$ when the two techniques were combined. ${ }^{14}$

Giovannini et al. ${ }^{15}$ defined the nCLE definitions for individual PCL subtypes in an endoscopic atlas published in 2014. These definitions were further refined and validated in a number of subsequent studies (Table 1).

The CONTACT studies compared nCLE videos to corresponding histopathological pictures. This pilot study defined a SCN by the presence of a superficial vascular network (Figure 1), which correlated to a dense and subepithelial capillary vascularisation, as seen on pathology. In the second part of the study, 66 images from 31 patients were shown to a group of experts. They found the definition to have an accuracy of $87 \%$, sensitivity of $69 \%$, specificity of $100 \%$, positive predictive value of $100 \%$, and negative predictive value of $82 \% .^{19}$
In the CONTACT 1 study, published by this group the following year, new nCLE criteria were described for MCNs, pseudocysts, and pancreatic neuroendocrine tumours. Mucinous lesions were differentiated, with an IPMN being defined by the presence of papillary projections (Figure 1) whereas MCNs were identified by a thick grey line. Bright uniform particles in clusters (likely representing macrophages) against the dark background were indicative of a pseudocyst. Furthermore, black neoplastic cells in clusters with white fibres were suggestive of a cystic neuroendocrine tumour. In a retrospective validation study, these criteria had a diagnostic specificity of $>90 \%$ for mucinous cysts and $100 \%$ for non-mucinous cysts. ${ }^{20}$

Kadayifci et al. ${ }^{21}$ explored the diagnostic utility of nCLE in mucinous cysts. They visualised typical features of mucinous cysts in eight out of 12 (66\%) cases. The superficial vascular network was observed in two out of three patients with a SCN. Sensitivity, specificity, and diagnostic accuracy of epithelial structures in detecting a mucinous cyst were $66 \%, 100 \%$, and $80 \%$, respectively.

In the CONTACT 2 trial, 202 patients were recruited. Of these individuals, 78 had PCLs with a pathology-based diagnosis (53 premalignant and 25 benign PCLs). nCLE was conclusive in 71 of the 78 cases (91\%). The sensitivity and specificity of nCLE for the diagnosis of SCN, mucinous PCL, and premalignant $P C L$ were all $\geq 95 \%$ (confidence interval: 85-100\%). In mucinous lesions, the area under the receiver operating characteristic curve was significantly better for nCLE than for carcinoembryonic antigen of $>192 \mathrm{ng} / \mathrm{mL}$ (0.98 versus $0.81 ; \mathrm{p}<0.01$ ) or EUS morphology (0.98 versus $0.82 ; p<0.05) .^{17}$

Krishna et al. compared in vivo and ex vivo images to final pathology in 10 patients to further define nCLE subtype features. Final definitions were similar to those proposed by the CONTACT group. 15,17,21 The main difference in definitions was that SCN was defined by the presence of a fernlike pattern on nCLE. In this study, in vivo and ex vivo nCLE correlated with surgical histopathology in all cases. ${ }^{22}$ These definitions were validated in 49 patients, 26 with a pathology diagnosis. The sensitivity, specificity, and accuracy for diagnosing mucinous PCL were $94 \%, 82 \%$, and $89 \%$, respectively. ${ }^{23}$ 
Table 1: Clinical effectiveness of needle-based confocal laser endomicroscopy in identifying common mucinous and serous pancreatic cystic lesions.

\begin{tabular}{|c|c|c|c|c|c|c|c|c|}
\hline Study & Diagnostic aim & Cases (n) & $\begin{array}{l}\text { Sensitivity } \\
(\%)\end{array}$ & $\begin{array}{l}\text { Specificity } \\
(\%)\end{array}$ & $\begin{array}{l}\text { PPV } \\
(\%)\end{array}$ & $\begin{array}{l}\text { NPV } \\
(\%)\end{array}$ & $\begin{array}{l}\text { Diagnostic } \\
\text { accuracy of } \\
\mathrm{nCLE}(\%)\end{array}$ & $\begin{array}{l}\text { Diagnostic } \\
\text { yield of } \\
\text { cytology } \\
(\%)\end{array}$ \\
\hline \multicolumn{9}{|l|}{ Mucinous PCL } \\
\hline $\begin{array}{l}\text { Konda VJ et } \\
\text { al., }{ }^{13} 2013 \\
\text { INSPECT }\end{array}$ & $\begin{array}{l}\text { Neoplastic cystic } \\
\text { lesions (IPMN, MCN, or } \\
\text { cancer) }\end{array}$ & 66 & 59 & 100 & 100 & 50 & 71 & 26 \\
\hline $\begin{array}{l}\text { Nakai Y et } \\
\text { al.., }{ }^{14} 2015 \\
\text { DETECT }\end{array}$ & Mucinous cystic lesions & 30 & 80 & 100 & 100 & 80 & 89 & 3 \\
\hline $\begin{array}{l}\text { Krishna SG } \\
\text { et al..16 } 2017\end{array}$ & Mucinous cystic lesions & 29 & 95 & 94 & NR & NR & 95 & NR \\
\hline $\begin{array}{l}\text { Napoleon B } \\
\text { et al., }{ }^{17} 2019 \\
\text { CONTACT }\end{array}$ & Mucinous cystic lesions & $\begin{array}{l}206 \\
\text { ( } 78 \text { in } \\
\text { subgroup)* }\end{array}$ & 95 & 100 & 100 & 94 & 97 & NR \\
\hline $\begin{array}{l}\text { Keane MG et } \\
\text { al., }^{18} 2019 \\
\text { CONCYST }\end{array}$ & IPMN & 56 & 90 & NR & 96 & NR & 87 & 66 \\
\hline \multicolumn{9}{|l|}{ Serous PCL } \\
\hline $\begin{array}{l}\text { Napoleon B } \\
\text { et al.,17 } 2019\end{array}$ & $\mathrm{SCN}$ & $\begin{array}{l}71 \\
\text { (subgroup)* }\end{array}$ & 95 & 100 & 100 & 98 & 99 & NR \\
\hline \multicolumn{9}{|l|}{ CONTACT } \\
\hline $\begin{array}{l}\text { Keane MG et } \\
\text { al., }^{18} 2019 \\
\text { CONCYST }\end{array}$ & $\mathrm{SCN}$ & 56 & 56 & NR & 55.6 & NR & 39 & 66 \\
\hline $\begin{array}{l}\text { Krishna SG } \\
\text { et al..16 } 2017\end{array}$ & $\mathrm{SCN}$ & 29 & 99 & 98 & NR & NR & 98 & NR \\
\hline $\begin{array}{l}\text { Napoleon B } \\
\text { et al., }{ }^{19} 2015 \\
\text { CONTACT }\end{array}$ & $\mathrm{SCN}$ & 31 & 69 & 100 & 100 & 82 & 87 & 36 \\
\hline
\end{tabular}

*Subgroup of those patients with a conclusive needle-based confocal laser endomicroscopy diagnosis were compared with final diagnosis.

IPMN: intraductal papillary mucinous neoplasm; MCN: mucinous cystic neoplasm; nCLE: needle-based confocal laser endomicroscopy; NPV: negative predictive value; NR: not reported; PCL: pancreatic cystic lesions; PPV: positive predictive value; $\mathrm{SCN}$ : serous cystic neoplasm. 
In a subsequent study by the same group, which included 29 patients, sensitivity, specificity, and accuracy for the diagnosis of mucinous PCL improved further to 95\%, 94\%, and 95\%, respectively. ${ }^{16}$ Recently, the same authors conducted a larger prospective study of 144 patients undergoing nCLE of a PCL. Sensitivity, specificity, and diagnostic accuracy for a mucinous lesion were 98\%, 94\%, and 97\%, respectively. nCLE was found to be more accurate in classifying mucinous and non-mucinous cysts compared with current clinical care $(p<0.001) .{ }^{24}$

The authors' group recently published the UK multi-centre experience of using nCLE in indeterminate PCL. The CONCYST trial showed EUS-nCLE had a superior diagnostic accuracy to cytology (76.8\% versus $71.0 \%$ ) but this did not reach clinical significance. More variation was seen in the diagnostic accuracy of individual PCL subtypes than had been recognised previously. The sensitivity of SCN was only $55.6 \%$ but was $90.0 \%$ for IPMN and $100.0 \%$ for pancreatic ductal adenocarcinoma. ${ }^{18}$

Recently, groups have also explored combining the use of nCLE with microforceps biopsy during the same EUS session. The diagnostic yield for each modality was $34.1 \%, 75.0 \%$, and 84.1\% for cytology, microforceps biopsy, and nCLE, respectively. When cytology, microforceps biopsy, and nCLE were combined, the diagnostic yield increased to $93.2 \%$ and led to a change in management in $52.3 \%$ of cases. NCLE led to a discontinuation in surveillance in $31.8 \%(p<0.05)$ and an additional $10.7 \%$ referred for surgery who otherwise would have been surveyed..$^{25}$

\section{Adverse Events}

The rate of associated adverse events, particularly acute pancreatitis, following EUSnCLE has been a concern. In the first pilot study by Konda et al. ${ }^{12}$ of EUS-nCLE using a prototype probe, technical challenges were encountered in 33.3\% and post-procedural pancreatitis occurred in $13.0 \%$ of patients with PCL. Cystic lesions appeared to be more susceptible to pancreatitis than solid masses. ${ }^{12}$ In the DETECT study, which used the $A Q$-Flex probe, rates of post-procedure pancreatitis of $7 \%$ were reported. This higher rate was attributed to the insertion of the cytascope in addition to the nCLE probe. ${ }^{14}$ In more recent, larger non-feasibility studies, rates of adverse events have fallen considerably and are similar to EUS FNA, with rates of pancreatitis 0.O$3.5 \%{ }^{16-19,21}$ This has largely been brought about by modifications to the technique, including pre-loading the probe into the FNA needle and limiting procedural time to less than 10 minutes. ${ }^{12}$

\section{Detecting Dysplasia and Prognostic Subtypes of Intraductal Papillary Mucinous Neoplasms}

Histologically, IPMNs are classified by type (gastric, intestinal, pancreatobiliary, or oncocytic) and level of dysplasia (low- or high-grade dysplasia or presence of invasive cancer). These details provide important prognostic information, which is usually only available after surgical resection. Recently, some groups have proposed that this information can be obtained by nCLE. In one study, four patients with different subtypes of IPMN were examined. EUS-nCLE showed characteristic finger-like projections with inner vascular core in all cases. Although the image patterns of the papillae for the gastric, intestinal, and pancreatobiliary subtypes were similar, in the oncocytic subtype, the papillae were thick and demonstrated a fine scale-like or honeycomb pattern, which correlated with pathology. ${ }^{26}$

A recent study also explored the feasibility of differentiating levels of dysplasia using nCLE. Cytology in cystic lesions is only diagnostic in approximately one-third of cases, even in high volume tertiary referral centres. ${ }^{8,27}$ In a recent study, which conducted a post hoc analysis of patients who had nCLE in resected IPMN, increased papillary epithelial width and darkness were found to have a sensitivity of $90 \%$ and $91 \%$, respectively, for detecting high-grade dysplasia or adenocarcinoma. ${ }^{24}$ However, it is known that there is heterogeneity in the level of dysplasia within the epithelium of an IPMN. ${ }^{28}$ Potentially, an area of high-grade dysplasia could therefore be missed during an nCLE examination because there is an area of the cyst that is inaccessible and therefore not imaged (e.g., behind the needle). However, these initial findings offer a potentially promising way of differentiating lowrisk lesions, which could be surveyed in order to avoid surgical resection. These findings will need to be validated in larger studies before being employed more widely in clinical practice. 


\section{Intraobserver Agreement}

Intraobserver agreement (IOA) between endosonographers has provided heterogeneous results in EUS-nCLE. IOA was first explored in the CONTACT studies. IOA was reasonable,with $\mathrm{K}=0.72^{20}$ and for SCN $\mathrm{K}=0.77 .^{19}$ In a further study by Karia et al., ${ }^{29}$ de-identified nCLE video clips were reviewed by six endosonographers at five institutions. The $\mathrm{k}$ statistics were low for individual imaging features, with a mean accuracy of only 46\% (range: 20-67\%). The low accuracy rates were attributed to poor image quality and the effect of the endoscopist individual learning curve. IOA and intraobserver reliability (IOR) have also been assessed for differentiating mucinous from non-mucinous cysts and were found to be $\mathrm{K}=0.67$ and $\mathrm{K}=0.78$, respectively. ${ }^{23}$

Recently, IOA and IOR was assessed in six endosonographers (each of whom had performed nCLE >30 times). They were asked to review the nCLE images of PCLs from 29 patients. The overall sensitivity, specificity, and accuracy for the diagnosis of mucinous PCL were 95\%, 94\%, and 95\%, respectively. IOA and IOR were also considerably higher, being $\mathrm{k}=0.81$ and $\mathrm{K}=0.86$, respectively, supporting that there is a learning curve to image interpretation in nCLE. ${ }^{17}$

\section{Cost-Effectiveness}

nCLE is an expensive technology, with high initial outlay costs for the laser scanning units, as well as ongoing probe costs per procedure, which are over and above the cost of an EUS examination. However, as described above, the device has potential to improve the reliability of confidently differentiating benign cysts such as SCN. This information can allow patients to be discharged from long-term surveillance and reduce the number of patients undergoing unnecessary operations. Thus, there is clear potential for healthcare cost savings. The members of the CONTACT study group conducted a retrospective health economic analysis for 209 nCLE cases conducted in France. They found nCLE over EUS FNA alone led to substantial changes in management for $28 \%$ of patients $(p<0.001)$, with a reduction in clinical costs of $13 \%$ in the public sector and $14 \%$ in the private sector. ${ }^{30}$

\section{DISCUSSION}

The international consensus guidelines on the management of IPMN recommend that an EUS is performed in all suspected IPMNs with worrisome features or cysts greater than $2 \mathrm{~cm}$, and when surveillance is advocated. ${ }^{31}$ Similarly, the American College of Gastroenterology (ACG) and European consensus guidelines recommended performing an EUS when findings are expected to change clinical management.,32 Although EUS can provide useful clinical information to influence the management of PCL, its utility is often limited by the ability to get sufficient fluid for analysis, the sensitivity of cytology for detecting dysplasia, and by current biomarkers (e.g., carcinoembryonic antigen) for accurately detecting mucinous lesions. ${ }^{27}$ nCLE allows real time imaging of the cyst wall and additional clinical information during the EUS examination. Therefore, it is an attractive emerging technology.

Multiple prospective studies have now described the utility of nCLE in indeterminate PCLs and in those PCLs with worrisome features. nCLE findings in cyst subtypes have been carefully correlated with pathological findings to provide accepted definitions. ${ }^{15}$ These definitions have then been validated in a number of multi-centre studies. The presence of a superficial vascular network or fern-like pattern is indicative of a SCN; papillary projections are suggestive of an IPMN; a dark band is representative of a $\mathrm{MCN}$; bright particles are characteristic of a pseudocyst; and dark areas are typical of malignancy. The most recent large multi-centre studies have shown that these criteria have a sensitivity of $>95 \%$ for detecting serous or mucinous lesions. ${ }^{16,17}$ However, there are some situations where nCLE has been found to be less effective, for example, when the epithelial lining has been denuded, which can occur as the cyst enlarges. In SCN, the presence of a superficial vascular network is indicative; however, in an oligocystic SCN, it may not be present. ${ }^{33}$ In an IPMN, it is known that the presence of inflammatory changes and dysplasia can be variable along the cyst wall. In addition, it is only possible to examine the adjacent wall of an IPMN with the probe, which could be a source of diagnostic discrepancies. Early studies have also demonstrated the potential of nCLE in detecting pathological subtypes of IPMN and 
levels of dysplasia. These findings are promising but require validation in larger studies. ${ }^{24,26}$

Rates of pancreatitis following nCLE are 0-13\%. ${ }^{14,19,26,34}$ Rates of adverse events have fallen substantially from the early nCLE studies (Table 1) and there appears to be a number of factors associated with increased rates of pancreatitis. The early nCLE studies backloaded the probe, which has the potential for more movement within the cyst. Therefore, this is no longer recommended. The DETECT study required longer needle access time because the procedure was combined with SpyGlass ${ }^{\mathrm{TM}}$ (Boston Scientific, Marlborough, Massachusetts, USA), cystoscopy, which prolongs the procedure and leads to a higher rate of post-procedural pancreatitis. ${ }^{14}$ Overall, any prolonged procedure time over 10 minutes or additional manipulation of the cyst should be avoided. ${ }^{14,26}$ Some of the adverse events are also potentially due to puncturing the cyst with a 19-gauge needle. In theory, if a probe compatible with a 22-gauge needle was available, this could make it easier to image cysts in the head of the pancreas and uncinate and potentially result in fewer adverse events; however, studies to formally evaluate safety and efficacy would be needed for verification.

Little is known about the learning curve in nCLE in PCL. There are two main aspects to the learning curve: the procedure and subsequent image interpretation. The technical aspects of the procedure are similar to an EUS FNA for experienced endosonographers; however, obtaining high-quality nCLE images and interpreting them takes more experience and training. ${ }^{16}$ The manufacturer provides online learning modules to support an endosonographer's image interpretation training on how to differentiate cyst subtypes, as well as offer in-room support with the device. Further studies are required to better define learning curves in this technique in order to inform future training programmes.

There has also been a growing interest in ways to improve nCLE interpretation and make it more straightforward for the endoscopist, such as through combining the technology with fluoroscopically-labelled markers and antibodies. In a study in Crohn's disease, labelled antibodies were administered topically in 25 patients, leading to the detection of intestinal membranebound TNF+ immune cells during confocal laser endomicroscopy. High numbers of membranebound TNF+ correlated with subsequent response to anti-TNF therapy and mucosal healing, demonstrating the potential for tailoring medical therapies. ${ }^{35}$ In the pancreas, the utility of this application has been demonstrated in swine. After injection of labelled antibodies against epidermal growth factor receptor and survivin into the pancreas with an EUS FNA needle, expression could be successfully detected with nCLE and could differentiate ductal from acinar cells. nCLE findings were confirmed on subsequent histology. ${ }^{36}$

\section{CONCLUSION}

EUS-nCLE is a promising technique, with improved diagnostic accuracy when compared to cytology and existing cyst fluid biomarkers. Recent studies have also demonstrated the potential of the technology to detect dysplasia, as well as cyst subtype, with reasonable accuracy. Overall, rates of associated adverse events are low. The technology is relatively expensive but may be cost effective in certain healthcare settings. Further work is needed to determine its place in diagnostic algorithms for PCL.

\section{References}

1. Laffan TA et al. Prevalence of unsuspected pancreatic cysts on MDCT. AJR Am J Roentgenol. 2008;191(3):802-7.

2. Spinelli KS et al. Cystic pancreatic neoplasms: observe or operate. Ann Surg. 2004;239(5):651-7.

3. Lee KS et al. Prevalence of incidental pancreatic cysts in the adult population on MR imaging. Am J Gastroenterol. 2010;105(9):2079-84.

4. Girometti $\mathrm{R}$ et al. Incidental pancreatic cysts on 3D turbo spin echo magnetic resonance cholangiopancreatography: prevalence and relation with clinical and imaging features. Abdom Imaging. 2011;36(2):196-205.
5. Adsay NV et al, "Intraductal neoplasm of the pancreas," Bosman FT et al (eds.), WHO Classification Of Tumors Of Digestive System (2010), Lyon: WHO Press.

6. Tanaka $\mathbf{M}$ et al. International consensus guidelines 2012 for the management of IPMN and MCN of the pancreas. Pancreatology. 
2012;12(3):183-97.

7. European Study Group on Cystic Tumours of the Pancreas. European evidence-based guidelines on pancreatic cystic neoplasms. Gut. 2018;67(5):789-804.

8. Keane MG et al. Clinical and radiological features that predict malignant transformation in cystic lesions of the pancreas: a retrospective case note review. AMRC Open Res. 2020;1:4.

9. Barresi L et al. Endoscopic ultrasound-through-the-needle biopsy in pancreatic cystic lesions: a multicenter study. Dig Endosc. 2018;30(6):760-70.

10. Kovacevic B et al. Initial experience with EUS-guided microbiopsy forceps in diagnosing pancreatic cystic lesions: a multicenter feasibility study (with video). Endosc Ultrasound. 2018;7(6):383-8.

11. Springer $\mathrm{S}$ et al. A combination of molecular markers and clinical features improve the classification of pancreatic cysts. Gastroenterology. 2015;149(6):1501-10.

12. Konda VJ et al. First assessment of needle-based confocal laser endomicroscopy during EUS-FNA procedures of the pancreas (with videos). Gastrointest Endosc. 2011;74(5):1049-60.

13. Konda VJ et al. A pilot study of in vivo identification of pancreatic cystic neoplasms with needle-based confocal laser endomicroscopy under endosonographic guidance. Endoscopy. 2013;45(12):1006-13.

14. Nakai $Y$ et al. Diagnosis of pancreatic cysts: EUS-guided, through-theneedle confocal laser-induced endomicroscopy and cystoscopy trial: DETECT study. Gastrointest Endosc. 2015;81(5):1204-14

15. Giovannini M et al. Endoscopic ultrasound guided confocal microscopy: atlas of cystic pancreatic lesions. Endosc Ultrasound. 2014:3(Suppl 1):S19-21.

16. Krishna SG et al. Needle-based confocal laser endomicroscopy for the diagnosis of pancreatic cystic lesions: an international external interobserver and intraobserver study (with videos). Gastrointest Endosc. 2017;86(4):644-54.e2.
17. Napoleon B et al. Needle-based confocal laser endomicroscopy of pancreatic cystic lesions: a prospective multicenter validation study in patients with definite diagnosis. Endoscopy. 2019;51(9):825-35

18. Keane MG et al. A prospective trial of CONfocal endomicroscopy in CYSTic lesions of the pancreas: CONCYST-01. Endosc Int Open. 2019;7(9):E1117-22.

19. Napoleon B et al. A novel approach to the diagnosis of pancreatic serous cystadenoma: needle-based confocal laser endomicroscopy. Endoscopy. 2015;47(1):26-32.

20. Napoleon B et al. In vivo characterization of pancreatic cystic lesions by needle-based confocal laser endomicroscopy ( $\mathrm{nCLE}$ ): proposition of a comprehensive nCLE classification confirmed by an external retrospective evaluation. Surg Endosc. 2016;30(6):2603-12.

21. Kadayifci A et al. Needle-based confocal laser endomicroscopy for evaluation of cystic neoplasms of the pancreas. Dig Dis Sci. 2017;62(5):1346-53.

22. Krishna SG et al. In vivo and ex vivo confocal endomicroscopy of pancreatic cystic lesions: A prospective study. World J Gastroenterol. 2017;23(18):3338-48.

23. Krishna SG et al. Validation of diagnostic characteristics of needle based confocal laser endomicroscopy in differentiation of pancreatic cystic lesions. Endosc Int Open. 2016;4(11):E1124-35.

24. Krishna SG et al. Endoscopic ultrasound-guided confocal laser endomicroscopy increases accuracy of differentiation of pancreatic cystic lesions. Clin Gastroenterol Hepatol. 2020;18(2):432-40.e6

25. Cheesman AR et al. Impact of EUSguided microforceps biopsy sampling and needle-based confocal laser endomicroscopy on the diagnostic yield and clinical management of pancreatic cystic lesions. Gastrointest Endosc. 2020:91(5):1095-104.

26. Kamboj AK et al. Confocal endomicroscopy characteristics of different intraductal papillary mucinous neoplasm subtypes. JOP.
2017;18(Suppl 2):198-202.

27. de Jong $\mathrm{K}$ et al. Endoscopic ultrasound-guided fine-needle aspiration of pancreatic cystic lesions provides inadequate material for cytology and laboratory analysis: initial results from a prospective study. Endoscopy. 2011;43(7):585-90.

28. Fischer CG et al. Intraductal papillary mucinous neoplasms arise from multiple independent clones, each with distinct mutations. Gastroenterology. 2019;157:1123-37.e22.

29. Karia K et al. Needle-based confoca endomicroscopy for pancreatic cysts: the current agreement in interpretation. Gastrointest Endosc. 2016:83(5):924-7.

30. Le Pen $\mathrm{C}$ et al. A health economic evaluation of needle-based confocal laser endomicroscopy for the diagnosis of pancreatic cysts. Endosc Int Open. 2017;5(10):E987-95.

31. Aad $G$ et al. Search for magnetic monopoles in sqrt[s]=7 TeV pp collisions with the ATLAS detector. Phys Rev Lett. 2012;109:261803.

32. Elta $\mathrm{GH}$ et al. ACG clinical guideline: diagnosis and management of pancreatic cysts. Am J Gastroenterol. 2018;113(4):464-79.

33. Alvarez-Sánchez MV, Napoléon B. New horizons in the endoscopic ultrasonography-based diagnosis of pancreatic cystic lesions. World J Gastroenterol. 2018;24(26):2853-66

34. Jais B et al. Serous cystic neoplasm of the pancreas: a multinational study of 2622 patients under the auspices of the International Association of Pancreatology and European Pancreatic Club (European Study Group on Cystic Tumors of the Pancreas). Gut. 2016;65(2):305-12.

35. Atreya $\mathrm{R}$ et al. In vivo imaging using fluorescent antibodies to tumor necrosis factor predicts therapeutic response in Crohn's disease. Nat Med. 2014;20(3):313-8.

36. Nakai $Y$ et al. In vivo visualization of epidermal growth factor receptor and survivin expression in porcine pancreas using endoscopic ultrasound guided fine needle imaging with confocal laserinduced endomicroscopy. J Physiol Pharmacol. 2012;63(6):577-80. 